\title{
Touching Annotations: A Visual Metaphor for Navigation of Annotation in Digital Documents
}

\author{
C.P.Bowers*, C.Creed, B.R.Cowan, R. Beale \\ Human-Computer Interaction Centre, School of Computer Science, University of \\ Birmingham, Birmingham, B15 2TT, UK
}

\begin{abstract}
Direct touch manipulation interactions with technology are now commonplace and significant interest is building around their use in the culture and heritage domain. Such interactions can give people the opportunity to explore materials and artifacts in ways that would otherwise be unavailable. These are often heavily annotated and can be linked to a large array of related digital content, thus enriching the experience for the user. Research has addressed issues of how to present digital documents and their related annotations but at present it is unclear what the optimal interaction approach to navigating these annotations in a touch display context might be.

In this paper we investigate the role of two alternative approaches to support the navigation of annotations in digitised documents in the context of a touch interface. Through a control study we demonstrate that, whilst the navigation paradigm displays a significant interaction with the type of annotations task performed, there is no discernible advantage of using a natural visual metaphor for annotation in this context. This suggests that design of digital document annotation navigation tools should account for the context and navigation tasks being considered.
\end{abstract}

Keywords: Document navigation, touch interaction, affordance, interaction techniques, user study

\footnotetext{
* Corresponding author

Email addresses: c.p.bowers@cs.bham.ac.uk (C.P.Bowers), creedcpl@bham.ac.uk (C.Creed), b.r.cowan@cs.bham.ac.uk (B.R.Cowan), r.beale@cs.bham.ac.uk (R. Beale)
} 


\section{Introduction}

Arts and cultural organisations are increasingly making use of touch enabled interfaces to allow the general public to interact with precious and rare artefacts. These interfaces have the potential to provide intuitive and engaging experiences when interacting with digital proxies of artefacts or manuscripts where the original physical version is inaccessible. This includes situations where restrictions on the number of artefacts constrain scalability and where interactions tend to hold a risk to the user or the artefact (e.g. dangerous substances or rare/precious artefacts).

In particular, museums and libraries regularly scan ancient manuscripts to make it possible for people to interact with digital versions of these documents (e.g. Turning the Pages ${ }^{\mathrm{TM}}$ ). These interfaces can provide compelling physical affordances allowing users to browse through documents, work collaboratively, and make comments or raise discussion about the material in an intuitive and familiar manner (Geller, 2006; Terrenghi et al., 2007; Liesaputra and Witten, 2012). Cultural organisations are particularly interested in collecting user generated content from the general public and visualising this in a way that makes it easy to navigate and view the annotations of others.

Annotations added to these documents are especially important as they help the general public and scholars learn more about the history and content of the documents. For these historical documents, annotation plays a critical role in translation, interpretation and comprehension of the content of these documents since they are often written in ancient script that result in difficult and, in some cases, multiple interpretations. Annotations can be part of an ongoing dialog between multiple readers. As such annotations can be gathered over the entire lifetime of a document and can include many forms of content including initial elucidations by the original document author through to contemporary scholarly interpretations (Agosti et al., 2007).

The nature of historical documents tends to result in the generation of large numbers of annotations that consist of significant amounts of crossreferencing both to aspects of the document and to other annotations. In order to gain a full appreciation of a documents content these numerous annotations must be presented in an intuitive and easily accessible way without being overwhelming.

Traditionally annotations have consisted of notes hand-written directly onto the original page, appended to the page in the form of a physically 


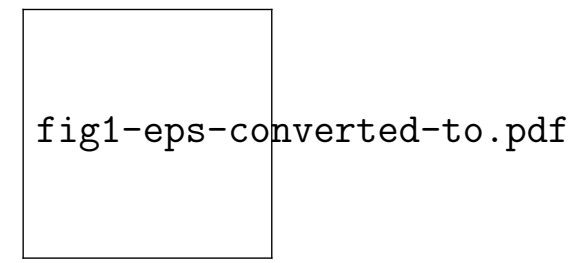

Figure 1: Use of thumbnails to support navigation between pages

attached note (e.g. Post-it ${ }^{\mathrm{TM}}$ notes) or held in a seperate document utilising some suitable form of cross-referencing (e.g. page \& line number). For digitised documents, whose origins predate many of the technical innovations in interweaving digital knowledge content through hyper-referencing, any enhancement must be appended to the original document. However, it remains unclear what the optimal interaction approach is in this context and in particular how users should navigate annotated documents on larger touch interfaces.

To better support public access to these documents we investigate the use of touch enabled interfaces for navigation of annotated documents. The software developed to support these activities typically focuses on the requirements of individual researchers working at a traditional desktop using a monitor, keyboard and mouse where users see scanned document pages on a screen and can navigate back and forth between pages by clicking on image thumbnails of other pages (Robinson, 2010). Figure 1 demonstrates the use of thumbnail images representing links to other document pages when viewing an historical text, whilst the current page of the text is displayed as a larger image in the centre.

A number of studies have looked specifically at document navigation using a traditional interface setup (i.e. ones using a mouse and keyboard), but less work has focused on whether these techniques are also effective on touch interfaces where users have the ability to directly manipulate and interact with the document and its annotations (Buchanan and Owen, 2008; Cockburn et al., 2006). The use of touch interfaces presents the opportunity to utilise real world metaphors and affordances that can create a more familiar interaction experience for users, yet it remains unclear whether this is an improved approach over more traditional navigation techniques.

In addition, although some effort has been made to explore the impact of touch interface designs on user performance metrics (Marshall et al., 2008; 
Shaer et al., 2011; Chiu et al., 2011), much of the research still focuses on reporting high level design solutions and qualitative evaluations. These studies, whilst providing valuable insights, do not readily identify the causal impact of interaction design choices on usability related metrics. Those quantitative studies that have been performed tend to focus on low-level interactions and manipulations (Cockburn et al., 2012). Consequently, little work in this area has been conducted to understand how the mode of interaction with annotations contribute causally to the usability of such interactions.

The work presented aims to investigate the usability of differing interaction designs in navigating digital versions of manuscripts in relation to their annotations. This paper explores how the mode of interaction with annotations within these documents impacts on user efficiency and usability judgement when navigating the content of a document using existing annotations. We start by covering related research on digital document annotation and then describe an empirical investigation comparing a real world interaction approach against a more traditional scrolling and thumbnail approach. We then provide a discussion of the significant statistical effects found along with suggestions for future research.

\section{Related Work}

Supporting the creation and navigation of annotations has been a fundamental feature of the development of digital document systems. We define the term annotation to mean any additional content that is added to the original document content. Annotations can be used to extend and enhance a document in a number of ways. They can be used to summarise/simplify complex content, allow cross-referencing of content, support the capture of alternative viewpoints, perspectives or discussions and better support search (Müller and Maurer, 2011).

An annotation can be a mix of any type of media including text, images, and videos. Most research on annotation in digitial documents has focused on the presentation of annotations and how the relationship between the annotation and the document content is supported (Chen et al., 2008; Pearson et al., 2009). Little focus has been placed on how to best support users in navigating existing annotations in relation to the document content (Kim et al., 2008). That is, how can users easily find document content that is of interest using the annotations in an efficient manner. 
Research has focused on the best approach for enabling users to add annotations to documents (Brush et al., 2001) rather than how these can support document navigation and how these impact usability related variables. For example research by Pearson et al. (2011) studied how a direct manipulation interface using lightweight digital annotation tools that reflected real world tools for physical documents (such as Post-it ${ }^{\mathrm{TM}}$ notes) support the act of annotating a document. The main aim of such research was to assess the nature of the annotation mechanism itself. The research presented in this paper looks to assess how tools for digital annotation support usability of document navigation, specifically in a touch interaction scenario.

Digital documents are increasingly being accessed, manipulated, and annotated digitally on touch devices such as smartphones, tablets, touch-enabled kiosks and touch tables. These interfaces afford new opportunities for working with annotated digital documents. In particular, touch interactions allow designers to create interfaces that utilise real world metaphors with which users are familiar such as being able to circle and highlight important areas of a document through digital ink (Bargeron and Moscovich, 2003; Golovchinsky and Denoue, 2002; Olsen et al., 2004) and also to represent annotations through coloured tabs attached to the side of the document (Buchanan and Owen, 2008).

However, research that has been conducted into navigating digital documents can be useful in understanding the techniques that might be best suited for navigating annotations in touch-based interfaces. A variety of different approaches have been examined and tested including space filling thumbnails (Cockburn et al., 2006), camera tilts (Guiard et al., 2006), multi-scale navigation (Appert and Fekete, 2006), following figure references (Buchanan and Owen, 2008), zoomable interfaces (Bederson, 2011), and sound cues (Eslambolchilar and Murray-Smith, 2006).

The use of scrolling to navigate documents is utilised in many popular applications (e.g. internet browsers, word processors, etc.) and as such has received much attention. Several researchers have observed that standard scrolling interfaces can have a negative impact on interaction by slowing down user progress due to the additional effort required to operate them (O'Hara and Sellen, 1997). Scrollbars can also have a detrimental impact with regard to a user's memory about the location of different elements in a document. This could negate the potential of their spatial memory to assist with navigation of a digital document (Cockburn et al., 2007).

A number of researchers have therefore examined how to enhance the 
scrollbar. Examples include the use of visualisations in the scrollbar (Byrd, 1999), navigation techniques for e-book readers (Chen et al., 2008), using techniques such as Speed Dependant Automatic Zooming (SDAZ) (Igarashi and Hinckley, 2000), and Rapid Serial Visual Presentation (RSVP) to replace scrolling with page flipping at high speeds (Sun and Guimbretière, 2005). Whilst positive benefits have been identified with scroll based approaches it remains unclear how well suited they are to touch screen interactions.

It must be noted that the hardware used in these interactions must also be considered. A number of related studies have also demonstrated the impact that the form and function of interaction hardware has upon the appropriateness of interaction design for navigation of documents. Jones et al. (1999) demonstrate that the size of a display can impact on appropriateness of interaction design with regard to scrolling and paging. Smith and schraefel (2004) describe how navigation performance can be improved by designing scrolling for touch interaction. However, no work to date has looked at navigation of annotation or compared new interaction techniques such as these with traditional interaction approaches on touch surfaces.

\section{Research Aims}

As discussed in the previous section interweaving digital knowledge content into existing digitised documents, in the form of digital annotation, is a growing challenge for heritage and cultural organisations wishing to provide access to such materials. Researchers have begun to address a number of these issues, in particular with regard to suitable presentation mechanisms

for annotation content. At the same time researchers have been investigating suitable interaction mechanisms to support digital document navigation. However, apart from Liesaputra and Witten (2012) examining how annotation can promote efficient and effective navigation via key word search, limited research has investigated how annotations impact upon navigation, especially using a direct navigation paradigm.

This work undertakes a comparative study of two common annotation presentation paradigms in the context of a touch enabled digital document interface. The aim is to identify how the manner in which they are presented might impact upon the efficiency of document navigation via those annotations. The first (Tab condition) will exemplify the use of real-world metaphors and natural affordances to facilitate navigation (Pearson et al., 2011; Liesaputra and Witten, 2012). The second (Scrollbar condition) will 


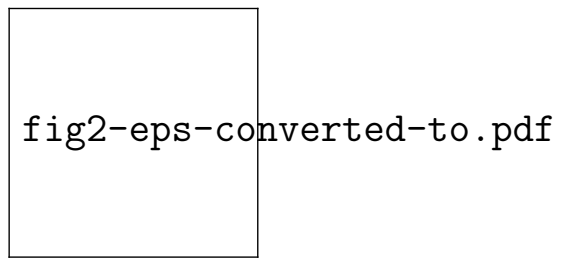

Figure 2: Visual metaphor of annotation in the form of tabs

draw from traditional document navigation interfaces (Buchanan and Owen, 2008; Cockburn et al., 2006).

\subsection{Tab condition}

The Tab condition utilised a real-world metaphor of annotation within a document by placing tabs along the fore-edge of the book (figure 2). This acts as a visual reference point for the annotation with the aim of providing a clear affordance in terms of navigating to an annotation.

The location of each tab on the fore-edge reflects the position of its representative annotation with respect to its position within the book. Tabs are arranged in order down the page, so that one tab does not occlude another. In addition, tabs on the left fore-edge of the documents facing pages reflect annotations that are prior to the currently selected annotation. Those to the right represent subsequent annotations following the current annotation within the document.

The book can be turned to the marked page by touching the tab, whereupon the relevant page is displayed along with the annotation and its tie indicating precisely what text it is attached to.

\subsection{Scrollbar condition}

The Scrollbar condition provides a navigation mechanism for the annotation that is more akin to navigation within a traditional desktop interface. In this case the tabs used in the previous condition are replaced with a scrollbar (figure 3).

The scrollbar utilises a scrolling list containing an overview of the annotation state of the document. The overview consists of a brief description of the annotation and a thumbnail of the relevant document page with annotated contents highlighted (figure 4). 


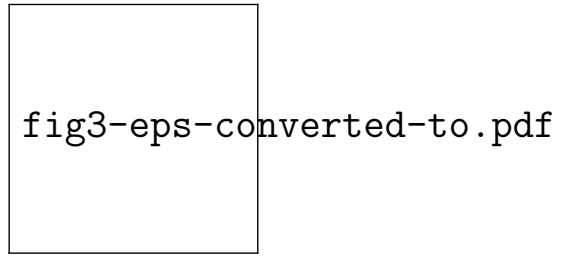

Figure 3: Navigation design utilising a scrollable list of annotations

scrollbar-eps-converted-to.pdf

Figure 4: Details of overview in scrollable list

The scrollbar is manipulated through the touch interface using vertical swipe gestures which are aligned so that the content moves with the users finger. If the user taps an annotation it opens the document at the location of the relevant annotation.

From the previous section we identify two factors that might influence the performance of annotation navigation: how the content of the annotation is presented and how the relationship between the annotation and the document content is supported. Two sets of navigation tasks are defined which explicitly account for these two factors. Three tasks require the annotation to be found based on the content of the document, such as annotations that refer to particular images or text. The other three tasks focus on the location of the annotation with respect to the contents of the document. The tasks are detailed in section 4.4. The tasks form two fundemental types of navigation and are used to undertake a comparitive task-based study. We aim to test the following hypotheses:

Hypothesis 1. There will be a significant interaction effect between task type and annotation navigation paradigm with respect to task efficiency.

Since the Scrollbar condition will provide more detail with respect to the content itself it is expected that users would be able to more quickly identify whether an annotation is relevant to the task if the task is related to the content of the document rather than the location. Additionally we hypothesise that users will be more efficient in the Tab condition where the 
task is dependent upon location of annotations relative to each other or to the document overall.

Hypothesis 2. Participants in the tab condition will rate the usability of the tab condition significantly differently to those in the scrollbar condition.

The visual metaphor using tabs to indicate the location of annotations more closely matches the affordances that a user might expect in the context of annotation within a physical document.

\section{Method}

\subsection{Participants}

33 participants took part in the study and were recruited from the body of staff and students at the University of Birmingham using an email request for participation. This email was posted to all departments across the university to gather a wide range of participants. A $£ 5$ gift voucher was given to each participant as an honorarium for participation.

\subsection{Conditions}

The experiment consisted of the two annotation navigation conditions for the document as described in section 3. These conditions make up the between participant variable Annotation Navigation in the analysis.

\subsection{Materials}

Although access to ancient documents in a culture and heritage context formed the initial inspiration for the research, we wished to generalise our findings more widely to the display of annotated documents using touchtables. The material chosen for the evaluation was Alice's Adventures in Wonderland by Lewis Carroll. This document is freely available as a set of high resolution page images. Annotations were generated by the authors to account for the experimental conditions. Due to the number of different versions of the book, each with different structures, it was felt that any familiarity a user might have with the story would not confound measurements of task performance made in this research.

The main document view is dominated by a centrally positioned image of the content of the document displayed in the form of facing pages. This accurately represents how the content of a physical book would appear to 


\begin{tabular}{|c|c|c|}
\hline No. & Task type & Task description \\
\hline 1 & \multirow{3}{*}{ Content } & Locate the first annotated image of Alice. \\
\hline 2 & & $\begin{array}{l}\text { Locate the first annotated image that includes } \\
\text { the rabbit. }\end{array}$ \\
\hline 3 & & Locate the third annotated image of Alice. \\
\hline 4 & \multirow[t]{3}{*}{ Location } & $\begin{array}{l}\text { Find the first annotation about the mad hatter } \\
\text { (around the middle of the book). }\end{array}$ \\
\hline 5 & & $\begin{array}{l}\text { Find the annotation about Alice eating a mush- } \\
\text { room (in the first third of the book). }\end{array}$ \\
\hline 6 & & $\begin{array}{l}\text { Find the annotation about Alice in the jury box } \\
\text { (in the final third of the book). }\end{array}$ \\
\hline
\end{tabular}

Table 1: The annotation navigation tasks presented to the participants

a user, leading to a more natural metaphor for interaction (Pearson et al., 2011).

Navigation of the document content was constrained under experimental condition to ensure that the only way for users to browse to the relevant location in the document was by the explicit annotation navigation condition. Therefore, no page turn gestures or buttons were provided.

\subsection{Tasks}

Two sets of navigation tasks are defined representing two fundamental type of navigation. The first set of three tasks require the annotation to be found based on the content of the document. The second set of three tasks focuses on the relationship between the annotation and the location of the relevent content within the document.

The 6 tasks presented to users are defined in table 1. In order to avoid ordering effects the task orders were counterbalanced across the participants using a balanced Latin Square approach. The tasks and related annotations were pre-determined and formed two distinct forms of Task Type.

\subsection{Dependent Variables}

Efficiency was quantified using response times for the tasks, measured as the time taken from the point at which the task was started by the user to the time at which they completed the task. 
Usability was measured using the Software Usability Scale (SUS) (Brooke, 1996). SUS has been used and researched in multiple studies (Bangor et al., 2008; Brooke, 1996; Lewis and Sauro, 2009) and consists of a 10-item scale that provides a subjective assessment of usability. In previous studies it has shown high internal reliability (Bangor et al., 2008). The questionnaire was administered using a Likert Scale from Strongly Disagree (1) to Strongly Agree (5). Negatively worded items were reverse scored and the scores for each item were summed to gain an overall usability score. This was then converted into a percentage. The scale therefore ranges from a minimum of 0 to a maximum of 100 .

\subsection{Procedure}

The experiment was conducted in a small room (approx. $3 \mathrm{~m} \times 2.5 \mathrm{~m}$ ) with controlled lighting to reduce reflection and eyestrain when interacting with the touchtable. The interface was presented on a 22 -inch multi-touch capable display positioned horizontally at a height of 36-inches.

On arrival participants were provided with a short overview of what they were required to do during the experiment. They were then asked to read through each of the annotations in the interface and get a general sense of the content contained within the annotations. This was an important initial task as it enabled participants to become familiar with the touch interface prior to undertaking the actual experiment. To avoid the interfering effects of users recalling the order, or content, of annotation a total of 12 separate annotations were applied to both text and illustrations within the document. Once participants had been through each of the annotations they informed the experimenter who then reset the interface and started the experiment.

At the start of the study participants were assigned to either the Tab or Scrollbar condition. A task was then presented to the user that involved them having to locate an annotation within the document. Once the participant was ready to start the task they tapped a "Start Task" button and began searching for the annotation. Once participants had started a task a "Finished Task" button was displayed at the top of the interface that they were asked to tap, once they had located the appropriate annotation. They were allowed to select and search through as many annotations as they wanted when performing their search (i.e. they did not have to guess the correct answer at the first attempt). When the "Finished Task" button was selected the next task was immediately displayed. This process was then repeated for the further tasks. 


\begin{tabular}{|c|c|c|c|c|}
\hline Annotation Navigation & Task Type & $\mathrm{N}$ & Mean (secs) & S.D. (secs) \\
\hline \multirow{2}{*}{ Tab } & Location & 16 & 29.20 & 17.60 \\
\cline { 2 - 5 } & Content & 16 & 34.75 & 17.49 \\
\hline \multirow{2}{*}{ Scroll } & Location & 17 & 36.07 & 26.68 \\
\cline { 2 - 5 } & Content & 17 & 17.65 & 8.40 \\
\hline \multirow{2}{*}{ Total } & Location & 33 & 32.74 & 22.66 \\
\cline { 2 - 5 } & Content & 33 & 25.94 & 15.97 \\
\hline
\end{tabular}

Table 2: Mean task times by conditions

After participants had finished all of the tasks they were then asked to complete the SUS questionnaire. Upon completion of the questionnaire they were debriefed and received their $£ 5$ gift card.

\section{Results}

\subsection{Task Efficiency}

The descriptive statistics for the sample in terms of amount of time taken to complete the task (in seconds) are displayed in table 2. The effect of Task Type (Content or Location Tasks - within participants) and Annotation Navigation (Tab or Scroll - between participants) on the time taken to complete the task (Efficiency) was analysed using a 2x2 mixed design ANOVA. There was no significant main effect of Task Type $[F(1,31)=2.68, p>0.05]$ or Annotation Navigation $[F(1,31)=0.97, p>0.05]$ on the time participants took to complete the tasks.

In support of our first hypothesis we observed a significant interaction between Task Type and Annotation Navigation on the time taken to complete the tasks $[F(1,31)=9.29, p<0.01]$. The interaction is presented graphically in Figure 5.

In order to explore this interaction effect further a paired samples ttests were performed for each Annotation Navigation condition. In the Tab condition there was no significant difference in efficiency between the Content $(M=34.75, S . D=17.49)$ and Location $(M=29.20, S . D .=17.60)$ task conditions $[t(15)=0.995, p>0.05]$. This means our prediction that the Tab condition better supported navigation tasks that relate to annotation and their relative positions to other annotations, or the overall document, was unsupported. 


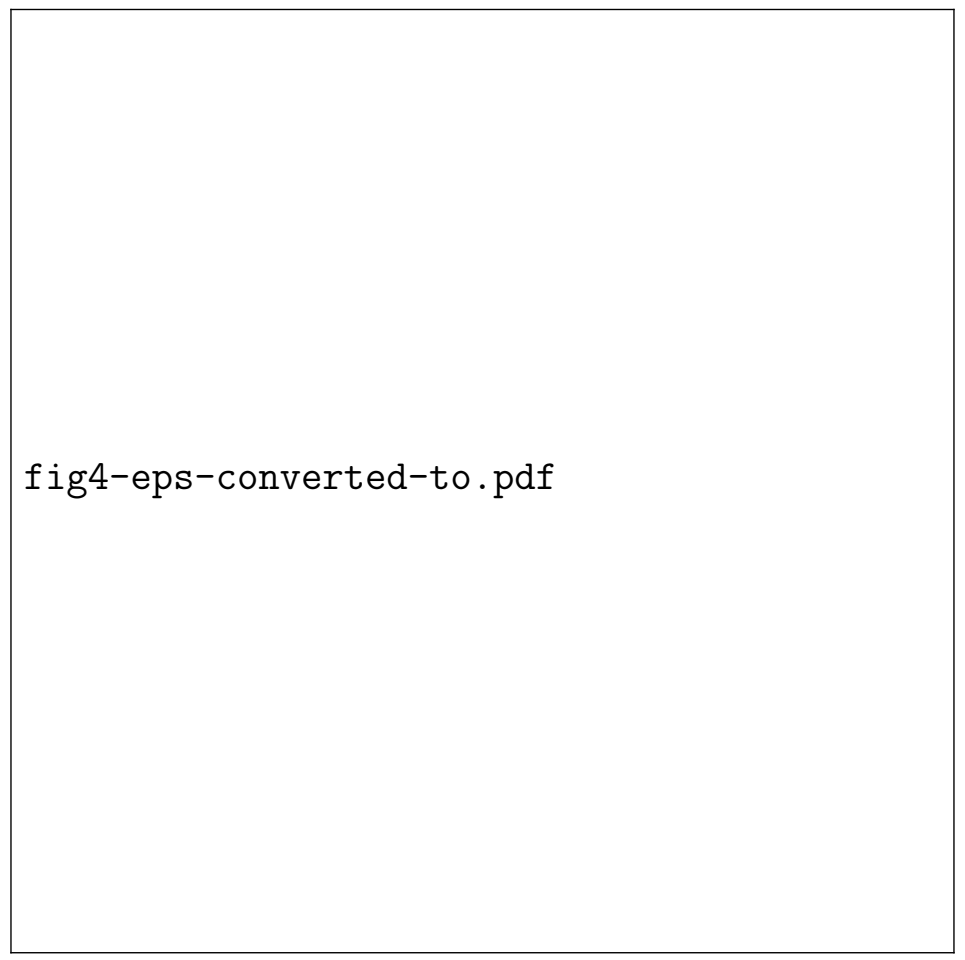

Figure 5: Mean \& Standard Error of task completion time for Annotation Navigation conditions by Task Type.

There was however a significant difference between the Content $(M=$ 17.65$, S.D. $=8.40)$ and Location $(M=36.07$, S.D. $=26.68)$ task conditions in the Scroll condition $[t(16)=-3.33, p<0.01]$. Participants completed the content tasks significantly faster than the location tasks when using the Scroll condition. This lends support for our hypothesis that there will be a significant difference in the Scroll conditions between the tasks.

It seems that the Scroll condition was felicitous for the content-based tasks compared to the location based tasks, whereas there was no significant impact of the Tab condition on the speed of task completion in the different task conditions.

\subsection{Subjective Usability}

Reliability analysis highlights that the Software Usability Scale (SUS) (Bangor et al., 2008) reached an acceptable level of reliability for a psycho- 


\begin{tabular}{|c|c|c|c|}
\hline Annotation Navigation & $\mathrm{N}$ & Mean (\%) & S.D. (\%) \\
\hline Tab & 16 & 85.78 & 12.57 \\
\hline Scroll & 17 & 83.09 & 7.73 \\
\hline
\end{tabular}

Table 3: Mean usability scores by conditions

metric scale (Cronbach $\alpha=0.74$ ) (Kline, 2000) although this is lower than alpha values reported in previous research (Lewis and Sauro, 2009). The descriptive statistics for the sample in terms of the usability scores are displayed in table 3 .

A Welch two samples t-test was used to analyse the effects of the Annotation Interface on participants usability rating. The Welch's t-test adjusts the degrees of freedom to correct for potential unequal variance between samples. There was no significant effect of Annotation Navigation on usability $[t(24.65)=0.74, p>0.05]$. Participants therefore did not rate the interfaces significantly differently in terms of usability. Our second hypothesis was therefore not statistically supported. The means and standard errors are displayed graphically in figure 6.

\section{Discussion}

Our results indicate that a comparison between a visual metaphor and a more traditional scroll based approach to document navigation in a touch interaction environment exhibits a significant interaction with the type of navigation task undertaken on task efficiency. People were significantly quicker completing the content tasks compared to the location task using the Scroll condition. There was no significant difference between task efficiency in the Tab condition.

When discussing navigation of annotation it is important to consider the context of use. In our case we utilise annotations that contain crossreferences relative to both the content of the document and location within the document.

For content-based tasks, where position of the annotation is provided with respect to other content of the document, it is clear that immediate access of the annotation content is important in supporting efficient navigation. In this case the scroll bar provided an easily accessible overview of each annotation. It therefore appears that some of the benefits of scrollbars 


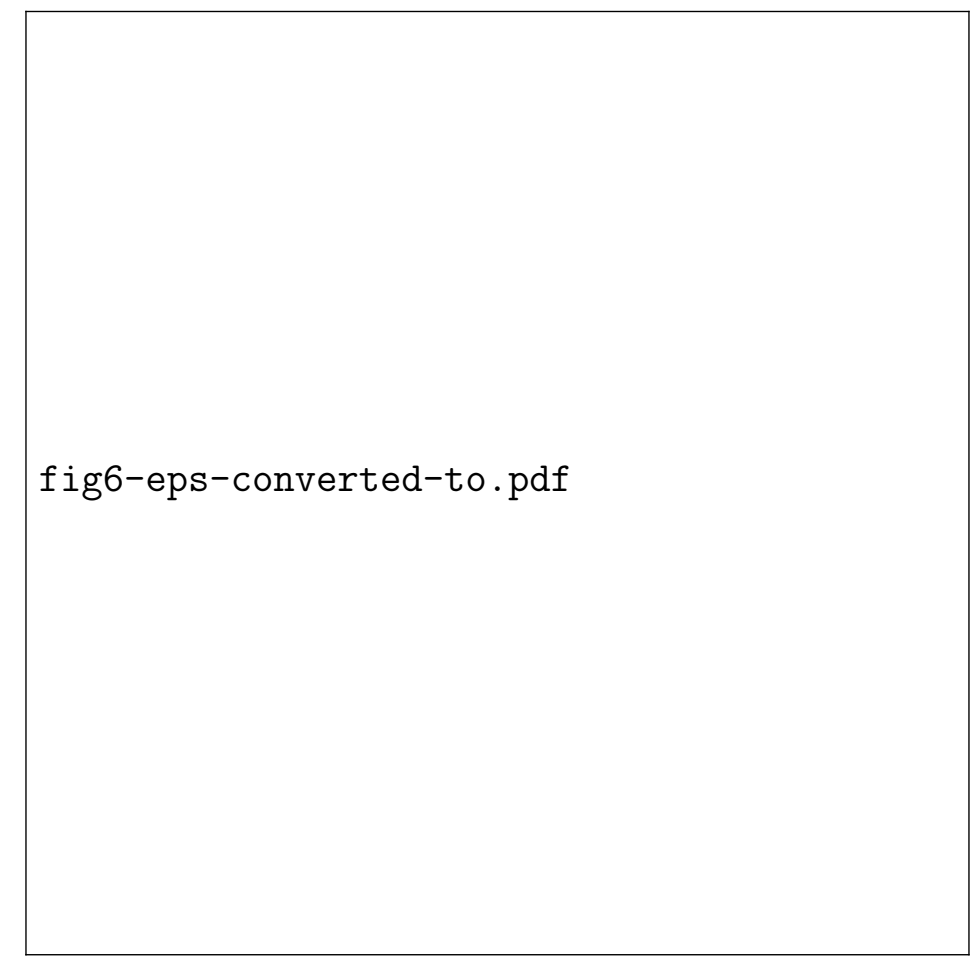

Figure 6: Mean \& Standard Error of Software Usabiity Scale score for Annotation Navigation conditions.

found in evaluations using traditional interfaces (Byrd, 1999; Chen et al., 2008; Igarashi and Hinckley, 2000; Sun and Guimbretière, 2005) also apply in touch-table interfaces (in the context of this study).

The benefit of a more natural tab based visual metaphor for annotation navigation in the context explored was less clear. The results indicate that participants in either annotation navigation conditions were able to navigate to the relevant part of the document with similar efficiency for location based navigation tasks. Additionally, there was no significant difference between the annotation navigation approaches in terms of user perception of usability. Our findings suggest that the Tab condition leads to a less efficient interaction within the content tasks than the Scroll condition but that there is relatively little difference between their efficiency in the location tasks.

We conclude that there is no discernable benefit from attempting to provide a more natural interface for navigation in this context and that more 
traditional models of navigation utilised in desktop interfaces are quite appropriate when modified to suit touch input. From our interaction it seems that a more natural interface in fact impacts negatively on efficiency when completing location based tasks. This is in contrast with seminal ideas on metaphor and affordances (Norman, 1988) that are typically adopted in an attempt to make interfaces more intuitive and simple to use.

This work demonstrates that context is all important when considering an appropriate interaction design for digital document navigation. Not only is the nature of the hardware platform of concern but perhaps more importantly the nature of the annotations being presented and how they are being used to navigate the document are important considerations.

\subsection{Limitations and Further Work}

This work attempts to fairly reflect the workflow of a research or nonexpert user navigating annotations within the context of a touch-table device within a controlled study. However, there are a number of limitations of this work that need to be considered.

One distinction between expert/non-expert users would be the length of time spent interacting with such systems. Our experiment did not identify the users experience with touch interactions before the experiment, although it can be assumed that the amount of interactions with a touchtable in this context is low. All participants were also given a practice session to acclimatise to the interface therefore the influence of such experience effects on task efficiency are likely kept to a minimum. Our navigation task and annotations were also relatively simple compared to the more complex cross-referencing that is prevalent in the annotations of ancient manuscripts (Baechler and Ingold, 2010; Müller and Maurer, 2011). Further work should aim to address this limitation and explore:

- The scalability of these approaches. This is especially important, as typically the number of annotations for a heavily researched manuscript will be very large.

- Support for semantic and hierarchical structure within the annotation such as topic or themed groupings. This will not only allow more complex annotation structure and cross-referencing but also assist in the retrieval of documents. 
A further study should determine whether a touch interface plays a significant role in the effect presented in this paper. Currently the study does not clarify whether these observed results are particular only to touch interfaces or observed in other platforms such as small touch screen devices (such as smart phones or tablet devices) or more traditional desktop environments. The constraints of these platforms in terms of size and the modality differences between desktop and touch interactions may impact specifically on the effect we have seen. It could be of value to identify and explore whether this effect reflects across platforms and modalities.

The reliability of the SUS scale also highlights a reduction in internal consistency in this context. It may be that when assessing such interactions as the ones being investigated in this research the SUS is not as reliable in measuring the construct of usability within a uni-dimensional scale as mentioned in other research. Although the questionnaire achieved a good reliability co-efficient, further research on touch table interaction should use the SUS to help identify whether this reduction in reliability is an issue of the interaction method being assessed. This would suggest refinement of the metric to more accurately reflect touchtable interactions could be needed.

Given the context of a touch-table environment it would also be valuable to investigate how navigation approaches impact on collaboration between users and how navigation techniques can support referencing between documents. Touchtables naturally provide an opportunity for collaboration and co-discovery, although recent research suggests users find it difficult to fully embrace collaborative working on touchtables (Marshall et al., 2008). It would be interesting to identify how navigation of annotation could be designed to impact and encourage co-discovery and how this could impact task efficiency and subjective usability. Recent research on interfaces used to support co-reading (Pearson et al., 2012) could be a useful starting point for such research.

Additionally further work should look to identify ways of developing interfaces to facilitate user generated content on such systems. Although we supplied all annotations for users to search in a cultural and heritage context one can envisage people potentially having a desire to contribute content and annotations that could be valuable to the interpretation of the document. Whether in a museum or academic collaboration context, designing an interface to facilitate contribution is something that could be of value in keeping annotations of these documents current and user led. 


\section{Conclusion}

To conclude, our research suggests that the type of navigation design impacts efficiency of task completion, yet this is dependent on the type of task being conducted. We found that there was no significant difference in task efficiency when completing the tasks using a more real world tab navigation condition. However a scroll navigation interface was felicitous when completing content-based tasks compared to location based tasks. Therefore when navigating annotations on touchtables, a scroll based interaction seems to bring benefits in content based task completion. As mentioned although we found this in a touchtable-based interaction, further research is needed to gather whether such an effect is relevant to other touch based platforms and other modalities of interaction. This paper also highlights the benefits of creating more natural affordances on touchtable based interactions, within an annotation navigation context, may not be universal.

\section{Acknowledgments}

This work was undertaken with support of JISC UK.

\section{References}

Agosti, M., Bonfiglio-Dosio, G., Ferro, N., 2007. A historical and contemorary study on annotations to derive key features for systems design. Int J Digit Libr 8, $1-19$.

Appert, C., Fekete, J.-D., 2006. Orthozoom scroller: 1d multi-scale navigation. In: Proceedings of the SIGCHI conference on Human Factors in computing systems. CHI '06. ACM, New York, NY, USA, pp. 21-30.

Baechler, M., Ingold, R., 2010. Medieval manuscript layout model. In: Proceedings of the 10th ACM symposium on Document engineering. DocEng '10. ACM, New York, NY, USA, pp. 275-278.

Bangor, A., Kortum, P. T., Miller, J. T., 2008. An empirical evaluation of the system usability scale. International Journal of Human-Computer Interaction 24 (6), 574-594.

Bargeron, D., Moscovich, T., 2003. Reflowing digital ink annotations. In: Proceedings of the SIGCHI conference on Human factors in computing systems. CHI '03. ACM, New York, NY, USA, pp. 385-393. 
Bederson, B. B., 2011. The promise of zoomable user interfaces. Behaviour and Information Technology 30 (6), 853-866.

Brooke, J., 1996. SUS-A quick and dirty usability scale. Usability evaluation in industry, 189-194.

Brush, A. J. B., Bargeron, D., Gupta, A., Cadiz, J. J., 2001. Robust annotation positioning in digital documents. In: Proceedings of the SIGCHI conference on Human factors in computing systems. CHI '01. ACM, New York, NY, USA, pp. 285-292.

Buchanan, G., Owen, T., 2008. Improving navigation interaction in digital documents. In: Proceedings of the 8th ACM/IEEE-CS joint conference on Digital libraries. JCDL '08. ACM, New York, NY, USA, pp. 389-392.

Byrd, D., 1999. A scrollbar-based visualization for document navigation. In: Proceedings of the fourth ACM conference on Digital libraries. DL '99. ACM, New York, NY, USA, pp. 122-129.

Chen, N., Guimbretiere, F., Dixon, M., Lewis, C., Agrawala, M., 2008. Navigation techniques for dual-display e-book readers. In: Proceedings of the twenty-sixth annual SIGCHI conference on Human factors in computing systems. CHI '08. ACM, New York, NY, USA, pp. 1779-1788.

Chiu, P., Liao, C., Chen, F., 2011. Multi-touch document folding: gesture models, fold directions and symmetries. In: Proceedings of the 2011 annual conference on Human factors in computing systems. CHI '11. ACM, New York, NY, USA, pp. 1591-1600.

Cockburn, A., Ahlström, D., Gutwin, C., Mar. 2012. Understanding performance in touch selections: Tap, drag and radial pointing drag with finger, stylus and mouse. Int. J. Hum.-Comput. Stud. 70 (3), 218-233.

Cockburn, A., Gutwin, C., Alexander, J., 2006. Faster document navigation with space-filling thumbnails. In: Proceedings of the SIGCHI conference on Human Factors in computing systems. CHI '06. ACM, New York, NY, USA, pp. 1-10.

Cockburn, A., Kristensson, P. O., Alexander, J., Zhai, S., 2007. Hard lessons: effort-inducing interfaces benefit spatial learning. In: Proceedings of the 
SIGCHI conference on Human factors in computing systems. CHI '07. ACM, New York, NY, USA, pp. 1571-1580.

Eslambolchilar, P., Murray-Smith, R., 2006. Model-based, multimodal interaction in document browsing. In: Renals, S., Bengio, S., Fiscus, J. (Eds.), Machine Learning for Multimodal Interaction. Vol. 4299 of Lecture Notes in Computer Science. Springer Berlin / Heidelberg, pp. 1-12.

Geller, T., 2006. Interactive tabletop exhibits in museums and galleries. Computer Graphics and Applications, IEEE 26 (5), 6-11.

Golovchinsky, G., Denoue, L., 2002. Moving markup: repositioning freeform annotations. In: Proceedings of the 15th annual ACM symposium on User interface software and technology. UIST '02. ACM, New York, NY, USA, pp. 21-30.

Guiard, Y., Chapuis, O., Du, Y., Beaudouin-Lafon, M., 2006. Allowing camera tilts for document navigation in the standard GUI. In: Proceedings of the working conference on Advanced visual interfaces - AVI '06. ACM, New York, New York, USA, pp. 241-244.

Igarashi, T., Hinckley, K., 2000. Speed-dependent automatic zooming for browsing large documents. In: Proceedings of the 13th annual ACM symposium on User interface software and technology. UIST '00. ACM, New York, NY, USA, pp. 139-148.

Jones, M., Marsden, G., Mohd-Nasir, N., Boone, K., Buchanan, G., 1999. Improving web interaction on small displays. Computer Networks 31 (11), $1129-1137$.

Kim, J.-K., Farzan, R., Brusilovsky, P., 2008. Social navigation and annotation for electronic books. In: Proceedings of the 2008 ACM workshop on Research advances in large digital book repositories. BooksOnline '08. ACM, New York, NY, USA, pp. 25-28.

Kline, P., 2000. A psychometrics primer. Free Association Books.

Lewis, J. R., Sauro, J., 2009. The factor structure of the system usability scale. In: Proceedings of the 1st International Conference on Human Centered Design: Held as Part of HCI International 2009. HCD 09. Springer, Berlin, Heidelberg, pp. 94-103. 
Liesaputra, V., Witten, I. H., 2012. Realistic electronic books. International Journal of Human-Computer Studies 70 (9), 588 - 610.

Marshall, P., Hornecker, E., Morris, R., Dalton, N. S., Rogers, Y., 2008. When the fingers do the talking: A study of group participation with varying constraints to a tabletop interface. In: Proceedings of the 3rd IEEE International Workshop on Horizontal Interactive Human Computer Systems. TABLETOP'08. IEEE, pp. 33-40.

Müller, H., Maurer, H., 2011. How to carry over historic books into social networks. In: Proceedings of the 4th ACM workshop on Online books, complementary social media and crowdsourcing. BooksOnline '11. ACM, New York, NY, USA, pp. 25-34.

Norman, D. A., 1988. The Psychology of Everyday Things. Basic Books.

O'Hara, K., Sellen, A., 1997. A comparison of reading paper and on-line documents. In: Proceedings of the SIGCHI conference on Human factors in computing systems. CHI '97. ACM, New York, NY, USA, pp. 335-342.

Olsen, Jr., D. R., Taufer, T., Fails, J. A., 2004. Screencrayons: annotating anything. In: Proceedings of the 17th annual ACM symposium on User interface software and technology. UIST '04. ACM, New York, NY, USA, pp. $165-174$.

Pearson, J., Buchanan, G., Thimbleby, H., 2009. Improving annotations in digital documents. In: Proceedings of the 13th European conference on Research and advanced technology for digital libraries. ECDL'09. Springer, Berlin, Heidelberg, pp. 429-432.

Pearson, J., Buchanan, G., Thimbleby, H., 2011. The reading desk: supporting lightweight note-taking in digital documents. In: Proceedings of the 15th international conference on Theory and practice of digital libraries: research and advanced technology for digital libraries. TPDL'11. SpringerVerlag, Berlin, Heidelberg, pp. 438-441.

Pearson, J., Owen, T., Thimbleby, H., Buchanan, G. R., 2012. Co-reading: investigating collaborative group reading. In: Proceedings of the 12th ACM/IEEE-CS joint conference on Digital Libraries. JCDL '12. ACM, New York, NY, USA, pp. 325-334. 
Robinson, P., 2010. Virtual manuscript room (vmr). Final report, Joint Information Systems Committee.

URL http://www.mingana. bham.ac.uk/

Shaer, O., Strait, M., Valdes, C., Feng, T., Lintz, M., Wang, H., 2011. Enhancing genomic learning through tabletop interaction. In: Proceedings of the 2011 annual conference on Human factors in computing systems. CHI '11. ACM, New York, NY, USA, pp. 2817-2826.

Smith, G. M., schraefel, m. c., 2004. The radial scroll tool: scrolling support for stylus- or touch-based document navigation. In: Proceedings of the 17th annual ACM symposium on User interface software and technology. UIST '04. ACM, New York, NY, USA, pp. 53-56.

Sun, L., Guimbretière, F., 2005. Flipper: a new method of digital document navigation. In: CHI '05 extended abstracts on Human factors in computing systems. CHI EA '05. ACM, New York, NY, USA, pp. 2001-2004.

Terrenghi, L., Kirk, D., Sellen, A., Izadi, S., 2007. Affordances for manipulation of physical versus digital media on interactive surfaces. In: Proceedings of the SIGCHI conference on Human factors in computing systems. CHI '07. ACM, New York, NY, USA, pp. 1157-1166. 